\title{
Operational limit of a planar DC magnetron cluster source due to target erosion
}

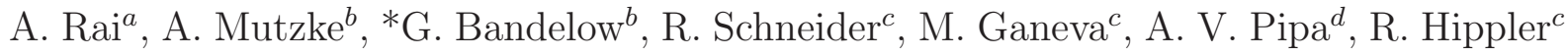 \\ ${ }^{a}$ University of Erlangen-Nuernberg, Institute of Science and Technology of Metals, \\ Martensstrasse 5, D-91058 Erlangen, Germany \\ ${ }^{b}$ Max-Planck-Institute of Plasmaphysics, EURATOM Association, D-17491 Greifswald, \\ Germany \\ ${ }^{c}$ Institute of Physics, Ernst-Moritz-Arndt-University Greifswald, Felix-Hausdorff-Str.6, \\ D-17489 Greifswald, Germany \\ ${ }^{d}$ Leibniz Institute for Plasma Science and Technology (INP Greifswald), Felix-Hausdorff-Str.Q, \\ D-17489 Greifswald, Germany
}

\begin{abstract}
The binary collision-based two dimensional SDTrimSP-2D model has been used to simulate the erosion process of a $\mathrm{Cu}$ target and its influence on the operational limit of a planar DC magnetron nanocluster source. The density of free metal atoms in the aggregation region influences the cluster formation and cluster intensity during the target lifetime. The density of the free metal atoms in the aggregation region can only be predicted by taking into account (i) the angular distribution of the sputtered flux from the primary target source and (ii) relative downwards shift of the primary source of sputtered atoms during the erosion process. It is shown that the flux of the sputtered atoms smoothly decreases with the target erosion.

* Corresponding author: Gunnar Bandelow (gstoppa@ipp.mpg.de

keywords: SDTrimSP, sputtering, magnetron
\end{abstract}




\section{Introduction}

Magnetron sputtering sources are the current work-horse of the sputter deposition technique of both metallic and non-metallic thin films on various substrates. The quality of the film depends on the amount of the deposition in terms of rate and coverage area. Both are determined from quantities such as the spatial, angular, and energetic distribution of both neutral gas and sputtered atoms that arrive at the substrate [1]. Magnetrons are also widely applied as a sources of free metal atoms in the gas aggregation nanocluster sources $[2-4]$.

A conventional balanced magnetron has a closed magnetic field configuration close to the cathode location. The magnetic field is parallel to the cathode surface. Secondary electrons which are emitted from the cathode due to ion bombardment are constrained by this magnetic field to move in a direction perpendicular to both the electric and the magnetic field ( $E \times B$ drift) [4] (see Fig. 1). This drift causes electrons to move parallel to the cathode surface in a direction perpendicular to the magnetic field. This $E \times B$ drift can be arranged to close on itself, forming a current loop of drifting secondary electrons which are trapped in a region close to the cathode. Eventually electrons lose their kinetic energy due to collisions with gas atoms or with other electrons and the net result is a dense plasma in this drift ring. Ions which are created in the drift region have a high probability of hitting the cathode which is close by. This results in even more production of secondary electrons and an extremely dense plasma. The erosion of the cathode is highest here and deep grooves can be eroded into the cathode, also known as the etch-track or race-track.

The formation of the erosion groove leads to continuous drift of the experimental conditions [2]. For better control of the experimental parameters detailed understanding of the erosion processes is required. In present work the influence of the target surface modifications on the flux of sputtered atoms is investigated. The main focus of this work is the simulation of the erosion process taking into account the angular distribution of the sputtered flux and the re-deposition process within the race-track region. 


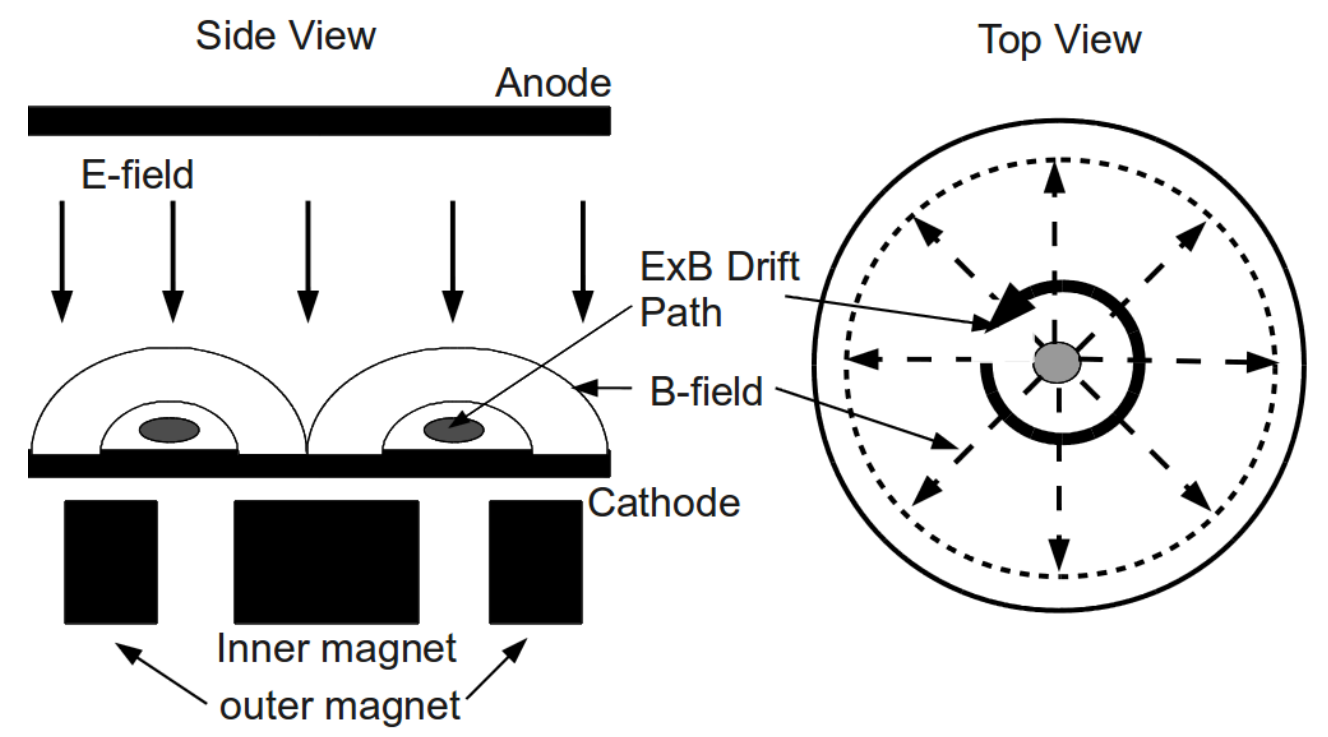

Fig. 1. Schematics of the magnetron discharge field configuration.

\section{Experiment}

Fig. 2 shows the schematic of the DC magnetron-based nanocluster source NC-200 produced by Oxford Applied Research. The detailed description of the source is provided elsewhere. [2] As a source of the sputtered particles conventional balanced magnetron equipped with a circular $\mathrm{Cu}$ target of $5 \mathrm{~mm}$ thickness and of 2 " diameter is used. The experimental conditions for the present work are: the discharge current is $0.4 \mathrm{~A}$, the discharge voltage is about of $300 \mathrm{~V}$, and the buffer gas (Ar) pressure in the magnetron chamber is $18 \mathrm{~Pa}$. The magnetron exhibits a current-voltage relationship of the form $I=k V^{n}$ where $n$ ranges from 7.1-7.6. The discharge voltage continuously decreases during the target operation ( $\sim 10 \%$ of the initial value) while the discharge current is kept constant.

Sputtered from the target free $\mathrm{Cu}$ atoms are used as a material for the cluster formation. The target lifetime is entirely defined by the erosion process. The erosion can be roughly characterized by the total energy dissipated in the discharge [2]. Thus, we consider the product of the discharge power and time as the target lifetime measured in kWh (kilo-watt hours). 


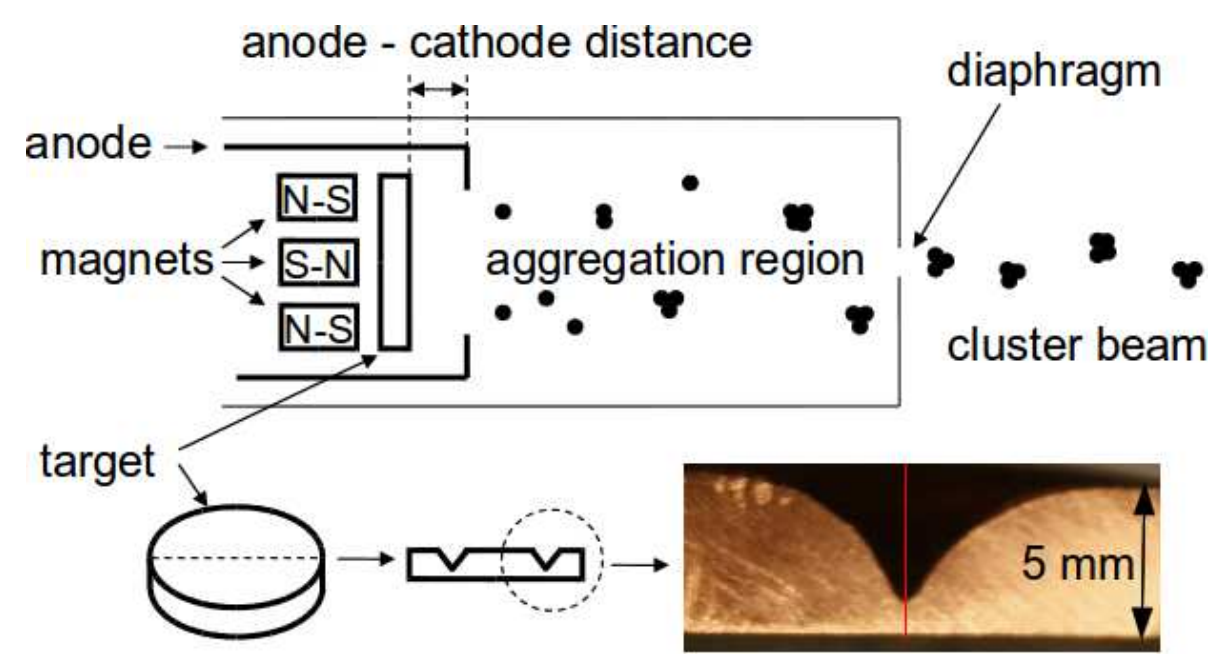

Fig. 2. General schematic of the magnetron cluster source. A circular Cu target of 2" in diameter is used as cathode which is biased by a negative DC voltage and the anode is grounded. The anode-cathode distance is $0.3 \mathrm{~mm}$. The photo shows a magnified cross-section of the race-track profile.

The size of clusters formed in the considered nanocluster source can be influenced by variation of magnetron power, pressure, temperature and length of the aggregation region. Quadrupole mass filter (QMF) has been used for measurement of the mass spectra of the produced cluster beam. Integration of the mass spectrum provides us the total cluster current shown in Fig. 3. The rapid growth of the total cluster current for the lifetimes below $0.1 \mathrm{kWh}$ is related to the pre-conditioning and not considered in the present work. For the lifetimes from 0.1 to $0.7 \mathrm{kWh}$ the total cluster current slightly decreases indicating a drift of the experimental condition with modification of the target surface. After $0.7 \mathrm{kWh}$ the cluster current abruptly drops down. The photo in Fig. 2 shows the cross-section of the target after the cluster formation is stopped. This corresponds to a groove depth of $3.8 \mathrm{~mm}$. Stop of the cluster formation long before the erosion groove reaches the target thickness was also observed for other experimental conditions and materials $[2,5]$.

\section{Model}

The primary source of free metal atoms needed to form the clusters in the magnetron cluster source is a $\mathrm{Cu}$ target being sputtered by the impinging $\mathrm{Ar}^{+}$ion. Therefore, in 


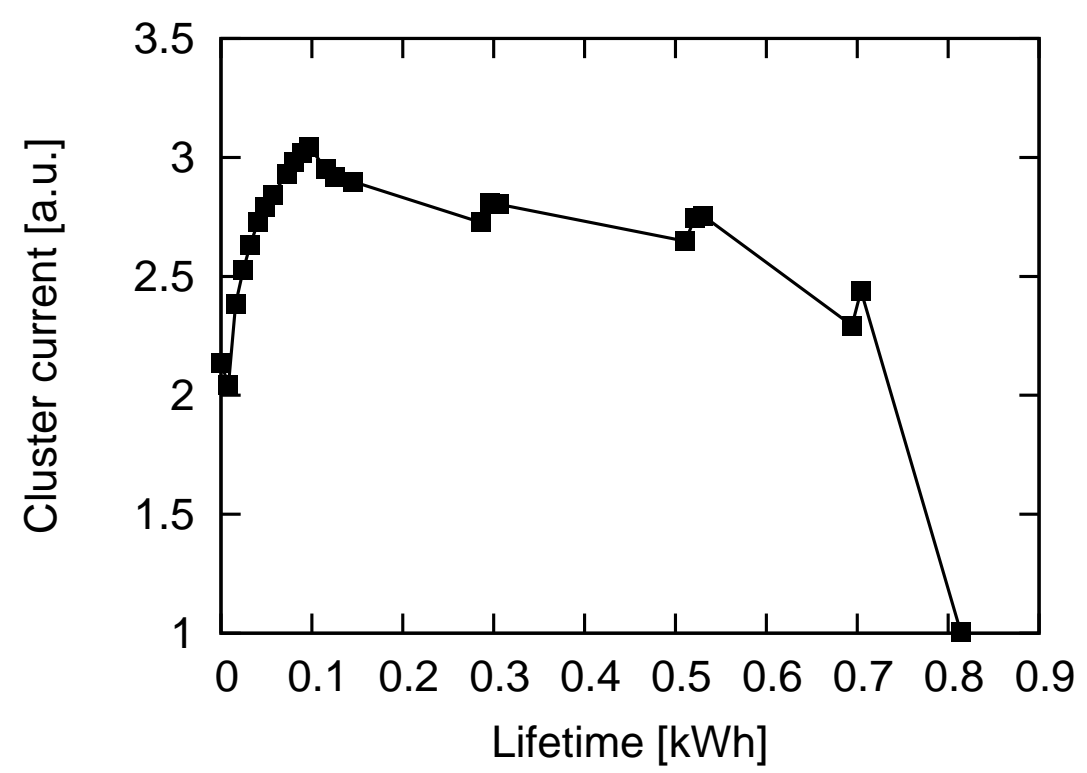

Fig. 3. Experimentally measured total cluster current as a function of target lifetime.

order to understand the variation in the measured cluster current and the change in free metal atom density, the morphological changes in the primary source (target) during its erosion should be studied. The process of sputtering determines the total amount of atoms released from the target. Once sputtered, the amount of sputtered flux measured at a given point is predominately a function of the geometry. The polar angular distribution of the sputtered particles can be expressed by $\cos ^{n} \alpha$, where $\alpha$ is the angle with respect to the surface normal. At low incident energies the distribution is under-cosine $(n<1)$ and at higher energies it becomes an over-cosine distribution $(n>1)$, which implies more intensity of sputtered particles at normal exit angles, $\alpha \approx 0$. Therefore, the sputtering efficiency combined with the geometrical constraints gives the density of the free atoms in the aggregation region which directly influences the measured cluster current. After being sputtered from the target, the released $\mathrm{Cu}$ atoms are transported towards the aggregation region and the sidewalls of the magnetron chamber. Their movement is inertial, with changes in velocity occurring only due to collisions. Part of the sputtered flux can be lost to the side walls of the chamber or get redeposited on the target itself.

The sputtered flux gives the total amount of atoms released from the surface. The density of the free atoms in the aggregation region can only be predicted by taking into account the angular distribution of the sputtered flux and the re-deposition process within the 
race-track region. The present work addresses this aspect of the sputtering of the $\mathrm{Cu}$ target for the given experimental conditions. Simulations are performed using SDtrimSP$2 \mathrm{D}$ to study the erosion process and the spatial profile of the sputtered flux is calculated. The sputtered flux of $\mathrm{Cu}$ is measured at diagnostic surfaces kept at 0, 1 and $10 \mathrm{~mm}$ above the $\mathrm{Cu}$ target. In particular the influence of the

(1) loss of the sputtered atoms to the chamber walls,

(2) shifting of the primary target source due to erosion, and

(3) redeposition of the sputtered atoms within the race-track region,

on the amount of sputtered material calculated at the diagnostic surfaces is analyzed. The transport of the sputtered particles and the cluster formation process in the aggregation region is not included in the model.

SDTrimSP $[6,7]$ is a binary collision code which simulates the interaction of energetic particles (e.g. Ar) with a target (e.g. $\mathrm{Cu}$ ) taking into account the dynamical changes within the target due to the bombardment and calculates various physical quantities like sputtering yield, energy and angular distribution of the sputtered flux and the depth profiles of different species in the target. SDTrimSP-2D is a 2-D extension of SDTrimSP [8], which, in turn, is a generalized version of the TRIDYN program [6], [9]. It can be run in static or dynamic mode (SD) on sequential or parallel systems (SP). SDTrimSP-2D uses a 2-D mesh to represent the surface morphology, the first dimension is the direction perpendicular to the macroscopic surface plane, and the second is in the direction parallel to that plane. This representation is sufficient to simulate the ion bombardment of surfaces with 2D micro-structure extended into the 3rd dimension. It is based on binary collision approximation like other codes of the TRIM family.

For a given ion beam with particle flux $\Phi$, the sputtering Yield $Y$ is defined as the ratio of the number of particles sputtered from the target, $N_{\text {sputter }}$, to the number of incident particles, $N_{\text {incident }}$. A target is defined by its length, depth and width corresponding to x, $\mathrm{y}$ and $\mathrm{z}$ directions, respectively, see Fig. 4 . 

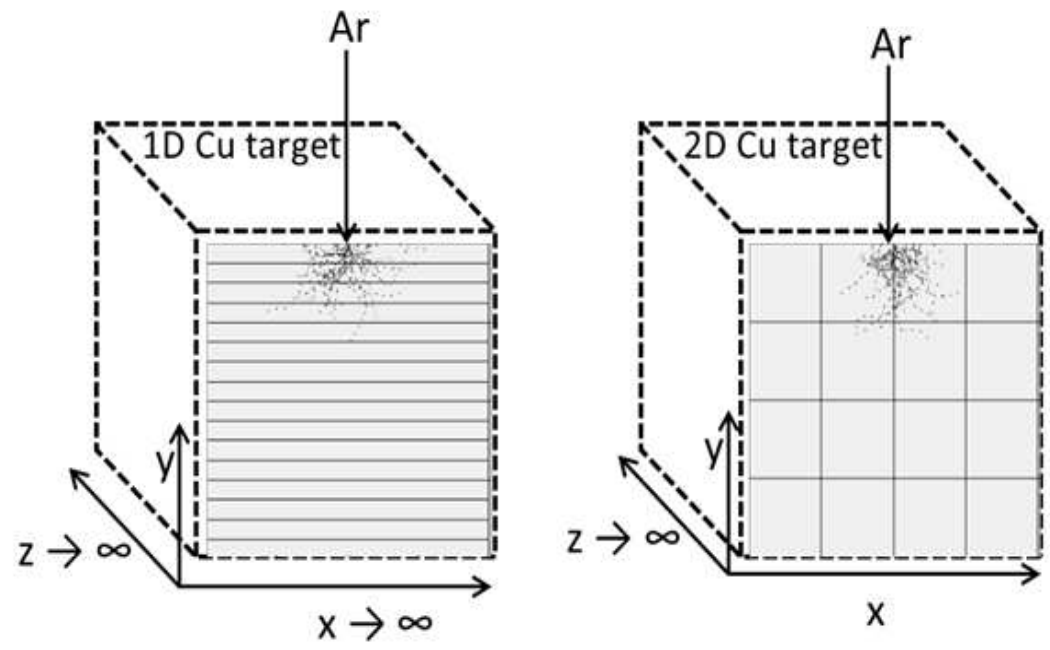

Fig. 4. Schematics of the 1D and 2D target geometry in SDTrimSP.

\section{Results}

The profile of the incident $\mathrm{Ar}^{+}$ions with $300 \mathrm{eV}$ energy is used as an input in the SDTrimSP-2D code. The spatial distribution of the incident $\mathrm{Ar}^{+}$ion density is assumed to be Gaussian. The width and the height of the distribution were chosen to get an agreement between the modeled and the experimental target erosion profiles (see curve e in Fig. 5). Fig. 5 shows the evolution of the surface with increasing fluence (the integral of the incident ion flux over time). The profile b seems to be similar to a Gaussian. With erosion development, the simulated profile start to deviate from the Gaussian shape and for profile e this difference becomes obvious. This emphasizes the effect of the redeposition process.

Depth distribution of the implanted $\mathrm{Ar}^{+}$ions in the target obtained using SDTrimSP-2D shows the maximum penetration depth of $\mathrm{Ar}^{+}$ions is $3 \mathrm{~nm}$ with atomic fraction of 0.02 . Therefore the implantation of Ar into the target is negligible.

Fig. 6 shows the target groove depth and total sputtering yield as a function of target lifetime. The SDTrimSP2D code calculates the sputtering for a given fluence of the incident ions. There is no explicit parameter corresponding to real time. However, the fluence 


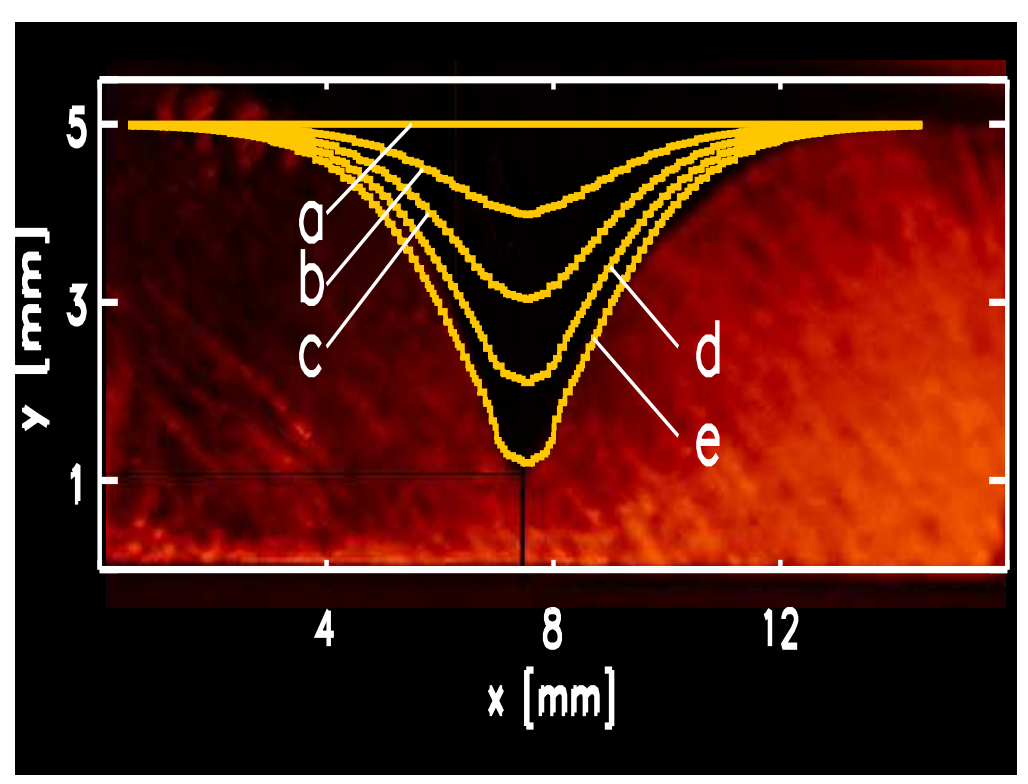

Fig. 5. Evolution of the surface. The experimental (red region) and simulated (yellow curve) target surface morphology as a function of fluence is shown.(a)-(e) the fluence is $(0,20,40,60,80) \times 10^{24} \mathrm{Ar}^{+}$ions $/ \mathrm{m}^{2}$.

can be related to the target lifetime as follows. The flux of the incident ions is proportional to the discharge current [10]. The dependence of the current on the voltage in the magnetron discharges is described as a power low. Hence, the discharge power can be controlled mainly by the discharge current while the discharge voltage is nearly constant. Therefore the product of the discharge power and time, so called target lifetime [2], is proportional to the fluence. Comparison of the simulated groove depth to the experimental one (Fig. 6) allows for a correlation between the fluence and the target lifetime.

The target groove depth can not be measured continuously during the experiment. It is done at the end of the experiment by cutting the target as shown in Fig. 2. The experimental data points for groove depth at 0.7 and $0.8 \mathrm{kWh}$ are obtained using different $\mathrm{Cu}$ targets. It was reported earlier that the use of different targets under similar experimental conditions gives rise to same qualitative results, but quantitatively they differ within a certain range [2]. This is thought to be the effect of different oxide layers on the target surface or due to the variation of the interelectrode distance.

The total sputtering yield, which is proportional to the flux of the free $\mathrm{Cu}$ atoms towards the aggregation region, monotonically decreases with the lifetime (see Fig. 6). Thus, the 
simulation of the present work shows failure of the assumption made in [2] that an abrupt drop of the cluster current at the end of the target lifetime is related to a rapid drop of the local sputtering yield due to large angles of the incident ions to the surface normal. Actually, such large angles were not reached in the present simulations. The sharp drop of the cluster current is likely related to specifics of the cluster formation processes. For a cluster formation, free $\mathrm{Cu}$ atom density must exceed a certain threshold, e.g. supersaturated vapor of metal particles should be reached [11]. Thus, a smooth decrease of free metal atom densities can lead to an abrupt drop of the cluster current.

In order to understand the effect of the geometrical constraints imposed due to the development of the erosion profile (well-shaped region in the target) on the loss of the sputtered particles to the wall and their redeposition on the target, sputtering characteristics of an eroded target are compared with that of an ideal flat $\mathrm{Cu}$ target.

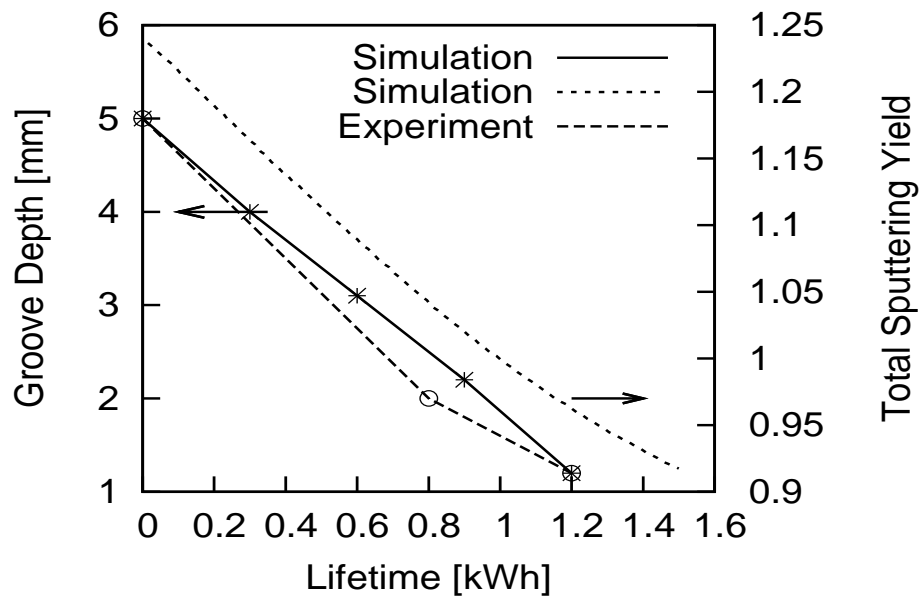

Fig. 6. Target groove depth and the total sputtering yield as a function of lifetime.

Fig. 7a and 7b show the profile of the sputtered flux from an ideal flat and an eroded $\mathrm{Cu}$ target, respectively. The $\mathrm{Cu}$ target shown in Fig. $7 \mathrm{~b}$ corresponds to the $3.8 \mathrm{~mm}$ deep race-track cross-section of the eroded target. The black curves in Fig. 7a and 7b show the profile of the incident Ar projectiles. The sputtering yield (red curve) from an eroded target (0.83) is much less than that from a flat $\mathrm{Cu}$ surface (1.23). In Fig. 7a, all the incident projectiles are normal to the surface $(\alpha=0)$ and therefore the released 
(a)

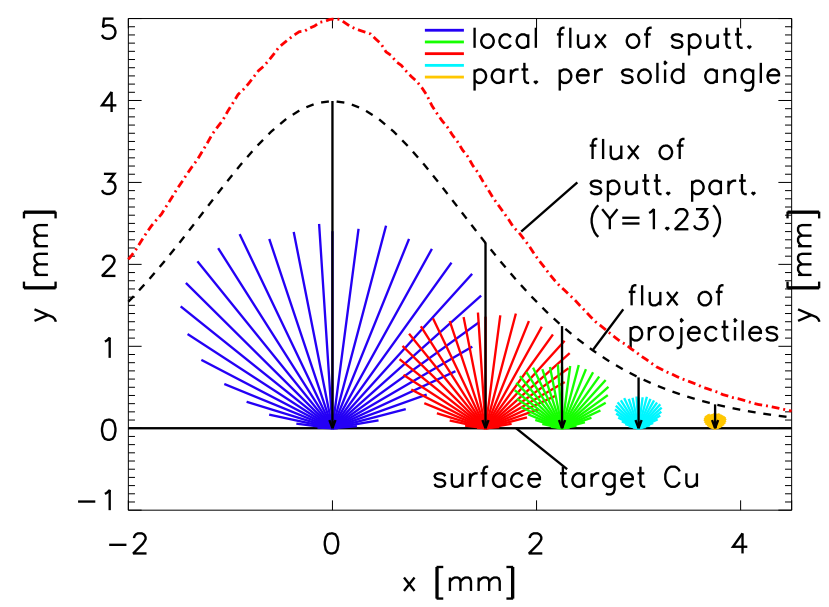

(b)

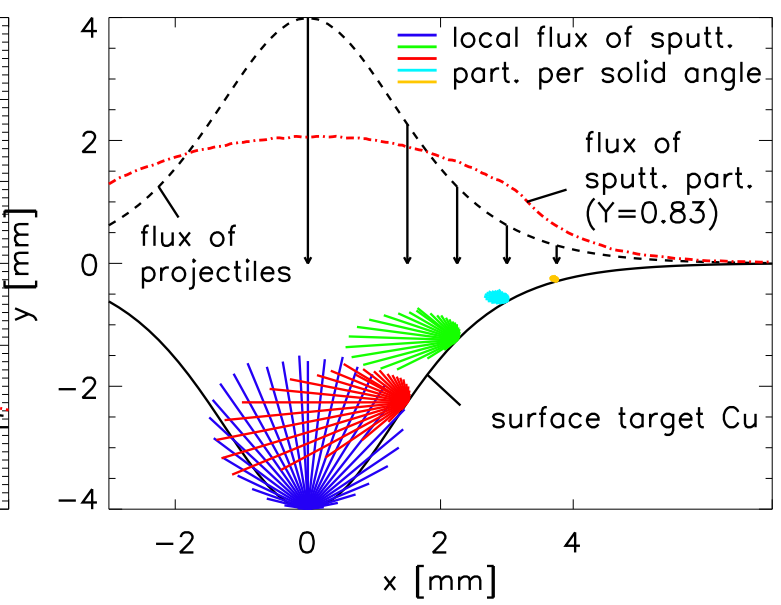

Fig. 7. Profile and direction of the incident $A r^{+}$ions (black curves and arrows respectively), corresponding sputtered flux envelop and the profile of the sputtered flux (red curve) for an ideal flat (a) and an eroded (b) target.

particles lie within a cosine envelope expected at $300 \mathrm{eV}$ [12]. The size of the envelops is proportional to the flux of the sputtered particles passing through it. In line with the profile of the incident $\mathrm{Ar}^{+}$ions, the maximum amount of $\mathrm{Cu}$ is sputtered at $x=0$. The angular distribution of the sputtered particles is identical at all the $\mathrm{x}$ locations, because it is determined by the angle of incidence of the projectile, which is same at all the $\mathrm{x}$ locations $(\alpha=0)$. Whereas, in Fig 7b all the projectiles do not hit the target normally $(\alpha \neq 0)$.

Fig. 8 shows the angular distribution of the sputtered $\mathrm{Cu}$ atoms for $\mathrm{Ar}^{+}$ions incident at $0^{\circ}$ and $49^{\circ}$ with respect to the initial surface normal for a flat (1D) and a eroded race-track profile $(2 \mathrm{D}) \mathrm{Cu}$ targets. For the $1 \mathrm{D}$ flat target the particles are sputtered around $\alpha=0$, i.e. normal to the surface. This is in accordance with the normal cosine distribution expected at this energy. Whereas, if the incident particles impinge at an angle of $49^{\circ}$ with the surface normal, it is seen that most of the particles are sputtered around $-100^{\circ}$. Looking at the final destination of the sputtered particles (Fig. 7b), it is clear that a major part of ejected particles (from $0<x<2.5 \mathrm{~mm}$ ) will get redeposited within the race-track well itself. The sputtering flux profile becomes much broader and there is a small bump around $x=3 \mathrm{~mm}$ corresponding to the particles which will land at the 


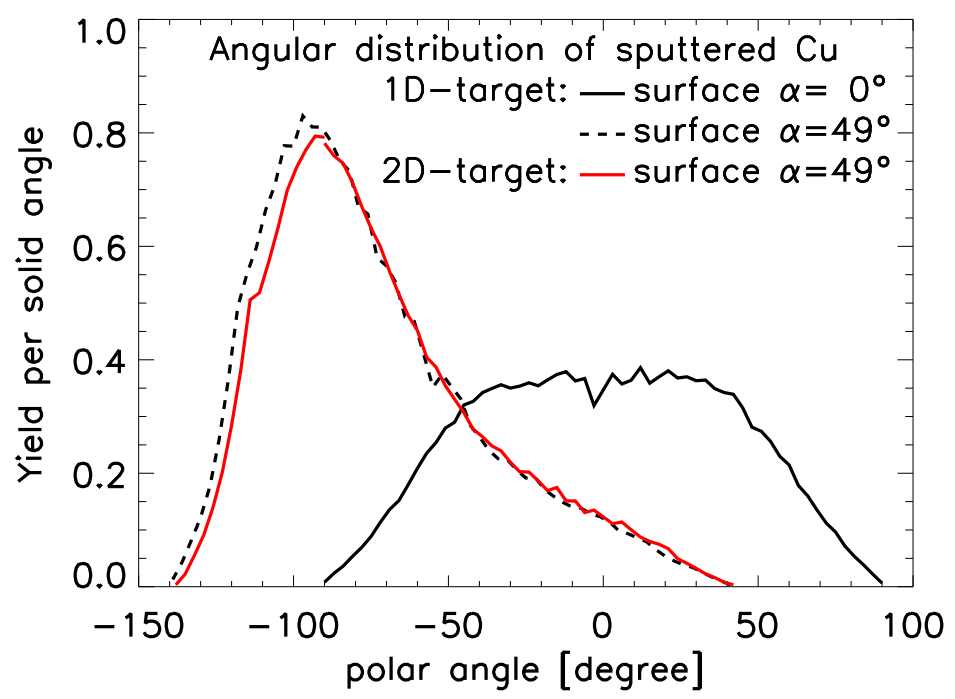

Fig. 8. Angular distribution of the sputtered $\mathrm{Cu}$ atoms for different angle of incidence.

side-walls of the experimental chamber. Due to redeposition and lowering of the overall sputtered flux, the contribution from the edges of the well is comparable to that from $x=0$.

In SDTrimSP-2D only neutral species and no collisions are included. Kolev et al. [1] have shown that the majority of the sputtered $\mathrm{Cu}$ flux are at low energy, $<5 \mathrm{eV}$. The self-sputtering of $\mathrm{Cu}$ is found to be negligible. Liu et al. [13] calculated the sticking probability of the $\mathrm{Cu}$ on a $\mathrm{Cu}$ surface as a function of incidence angle for various energies. They found out that for $\mathrm{Cu}$ with energy $<10 \mathrm{eV}$ the sticking probability is 1 , irrespective of the incidence angle. Using these two observations it can be stated that the sputtered $\mathrm{Cu}$ gets stuck/redeposited on the surface of the eroded well. This further supports the conclusion drawn using the SDTrimSP-2D simulation that redeposition plays a dominant role in the reduction of the sputtered flux from the primary source during the target lifetime.

In Fig. 9 the energy distribution of the sputtered particles from a flat and a race-track profile surface (now onwards mentioned as eroded target) as a function of the incident angle of the $\mathrm{Ar}^{+}$ions is shown. It is seen that in case of the eroded target there is an overall reduction in the sputtered flux and the whole energy spectrum shifts towards lower 

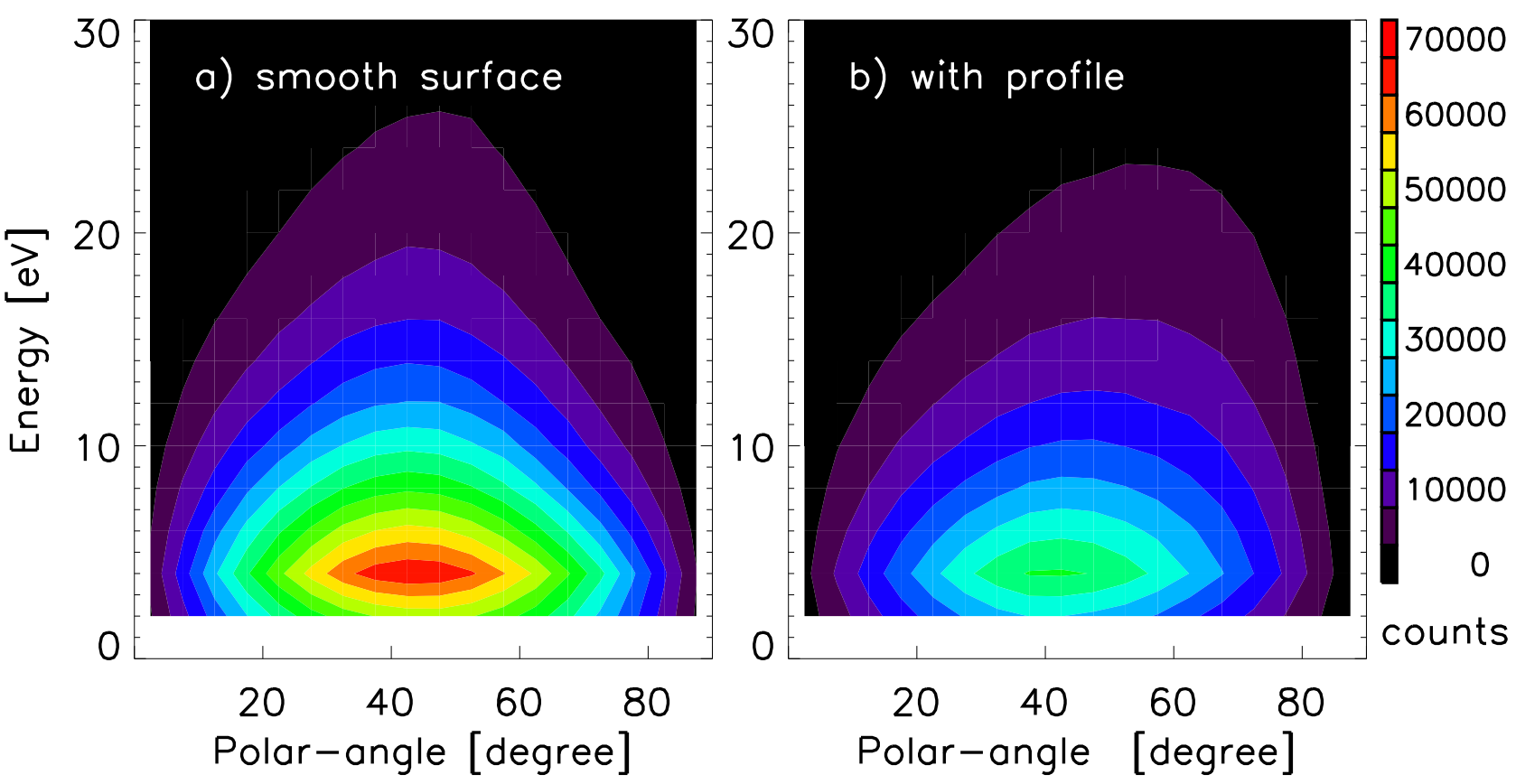

Fig. 9. Energy distribution of the sputtered particles measured at the diagnostics surface held at $0 \mathrm{~mm}$ above the initial geometrical surface in $\mathrm{y}$-direction for (a) flat surface and (b) for a surface with race-track profile.

energies. The main contributors to this reduced sputtered flux are low energy $(\sim 5 \mathrm{eV})$ particles sputtered from ions incident at $30^{\circ}-60^{\circ}$ angle with respect to the surface normal.

In order to understand this observation the dynamics of the collision cascade should be carefully looked into. As proposed by Biersack et al. [14], there are four processes leading to sputtering. When the sputtered atom receives its energy from the incident ion directly then it is called primary knock-on atom (PKA) otherwise if it is obtained via a target atom it is called secondary knock-on atom (SKA). Moreover, the momentum of the incident ion can be directed towards the target (ion-in) or out-of the target (ion-out). The relative contribution of all these processes depends on the energy, incident angle of the incident ion, the ratio of the projectile/target atom masses and the cathode (target) potential. The general observation is that the sputtering yield increases with increasing incidence angle of the projectile. For $\mathrm{Ar}^{+}$ions hitting a $\mathrm{Cu}$ target the maximum in sputtering yield is observed at $45^{\circ}[15]$, afterwards, an increase in the angle of incidence leads to more incident ions bouncing off the surface without enough transfer of energy and momentum 


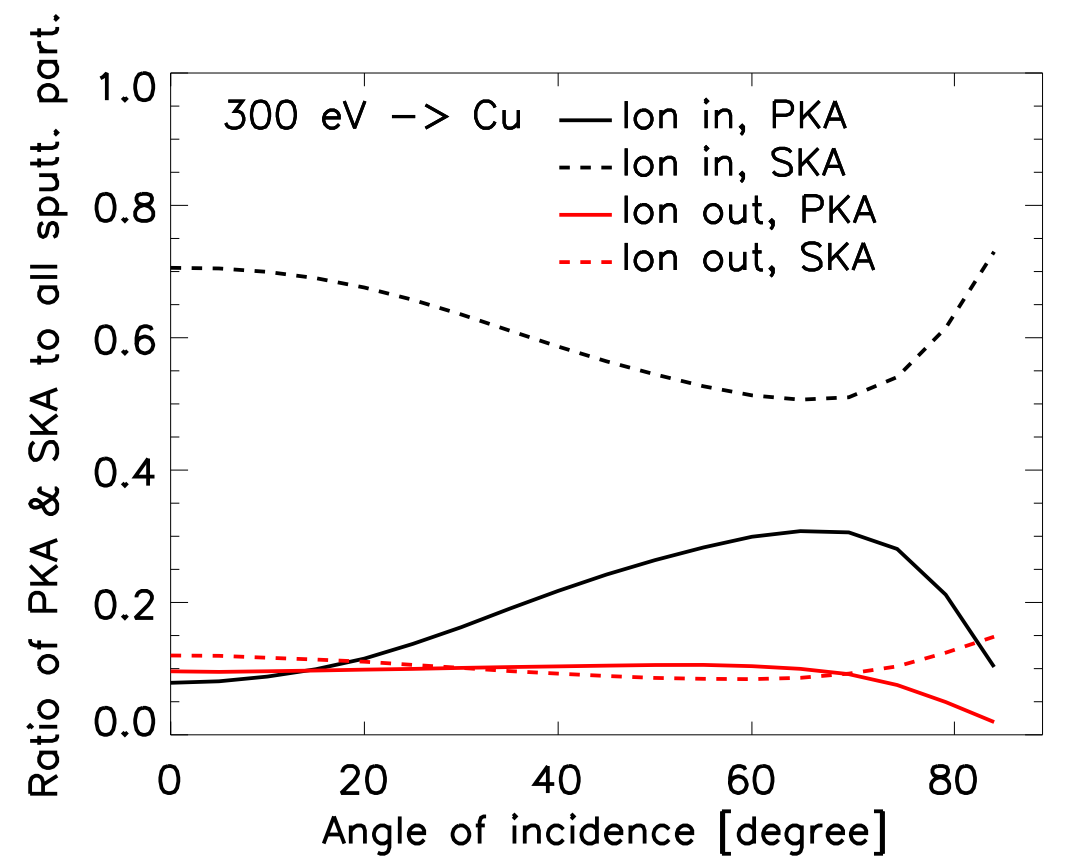

Fig. 10. Contribution of the four type of processes to the sputtering vs angle of incidence $\alpha$. resulting in a drop in the sputtering yield. Fig. 10 shows the relative contribution of the four main processes leading to sputtering. It is clearly seen that at lower angle of incidence where most of the energy and momentum is directed onto the target the main contribution for sputtering is coming from low energy recoils (ion-in SKA). Whereas, at higher angle of incidence a significant contribution comes from ion-in PKA as well. This is due to the fact that at higher angle of incidence the component of momentum into the target becomes smaller (though the total momentum and energy remains the same). The transfer of energy and momentum stays closer to the surface leading to higher probability of ion-in PKA generation.

Based on the energy of the sputtered $\mathrm{Cu}$ a back of the envelope estimate can be made 
about their mean free path and the influence of the collisions. The mean free path of a sputtered $\mathrm{Cu}$ atom with number density $\mathrm{n}$ is given by, $\lambda=(\sigma n)^{-1}$, with $\sigma$ being the cross-section for a given kind of the collisional process. For the magnetron discharge presented here, the typical density of the sputtered $\mathrm{Cu}$ atoms just above the cathode in the similar pressure range is $\sim 5 \times 10^{18} \mathrm{~m}^{-3}[1]$. There can be three collision possibilities for a sputtered $\mathrm{Cu}$ atom:

(1) elastic scattering of $\mathrm{Cu}$ atoms and ions from $\mathrm{Ar}$ atom: energy of the sputtered $\mathrm{Cu}$ atoms ranges from $1-20 \mathrm{eV}$ (see Fig. 9). For this energy range $\sigma$ is $2-1 \times 10^{-19} \mathrm{~m}^{2}$ [16], therefore $\lambda \sim 1-2 m$.

(2) electron impact ionization $\left(e^{-}+C u \rightarrow 2 e^{-}+C u^{+}\right)$: in the energy range 10-20 eV, $\sigma$ is $1-2 \times 10^{-20} \mathrm{~m}^{2}$ and $\lambda \sim 10-20 \mathrm{~m}$.

(3) neutral-neutral collision (collision between the sputtered $\mathrm{Cu}$ atom and the background Ar atoms): using the analytical approach used by Kolev et al. [1], in the energy range $1-20 \mathrm{eV}, \sigma$ is $1.4-0.5 \times 10^{-17} \mathrm{~m}^{2}$ and $\lambda \sim 1.4-4 \mathrm{~cm}$.

In the simulations presented here the sputtered flux of $\mathrm{Cu}$ is measured at the diagnostic surfaces kept at 0,1 and $10 \mathrm{~mm}$ above the $\mathrm{Cu}$ target (see Fig. 11). Since the shortest mean free path due to the neutral-neutral collisional is at least $1.4 \mathrm{~cm}$, it is justified to ignore their role in the presented simulations. However, during the cluster formation process in the aggregation region (12 cm long), effect of the collisions must be taken into account.

Fig. 11 shows the relative sputtered flux per unit length measured at various locations above the target for an eroded target and for a standard $\mathrm{Cu}$ target without erosion process. The inlet shows the location of the diagnostic surfaces kept at 0,1 and $10 \mathrm{~mm}$ above the initial target. Both the cases show a similar pattern, namely, the reduction in the sputtered flux as one moves away from the target. As the height of the diagnostic surface increases the profiles becomes broader and at $10 \mathrm{~mm}$ above the target the sputtered amount per unit length along the radius of the target is practically the same. The maximum amount is sputtered from the center of the race-track profile well. This result is in good agreement 


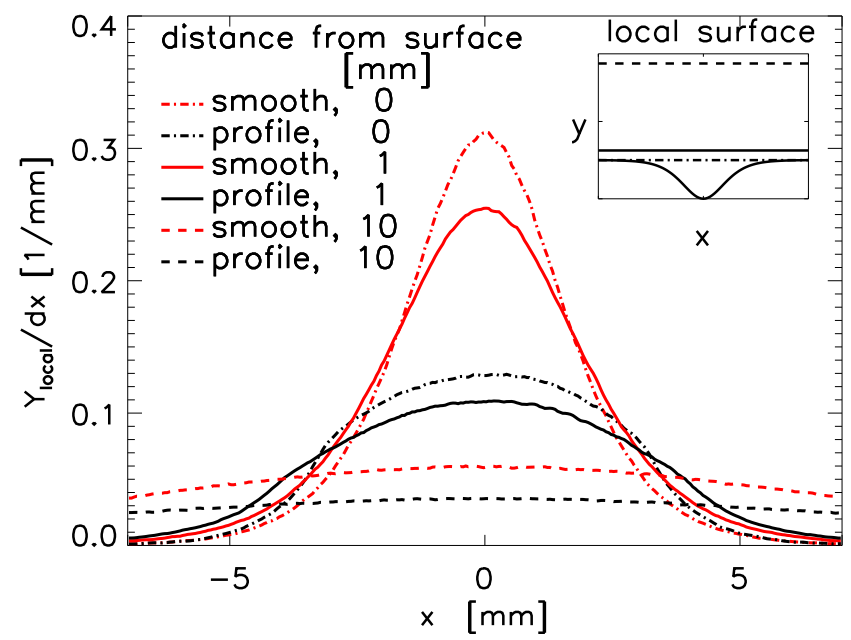

Fig. 11. Sputtered flux per unit length measured at various locations above the target.

with Kolev et al. [1] where they measured the sputtered flux as a function of the targetsubstrate separation.

The sputtering source can be treated as a point source with the intensity of the sputtered particles (sputtered amount per unit area) being proportional to the square of the distance from the point source $\left(1 / r^{2}\right.$ dependence). The reductions in the sputtered flux follows the expected $1 / r^{2}$ dependence. It was shown in Fig. 5 that with the increase in fluence (equivalently the target lifetime) the center of the race-track profile well becomes deeper due to erosion. Since the maximum of the sputtered flux comes from the center of the race-track profile well, this implies that during the erosion process, the location of the primary source of the $\mathrm{Cu}$ atoms from the cluster source moves away from the aggregation chamber. This leads to the reduction of the $\mathrm{Cu}$ atom density in the central region of the aggregation chamber and eventually contributes to the drop of the measured cluster 
(a)

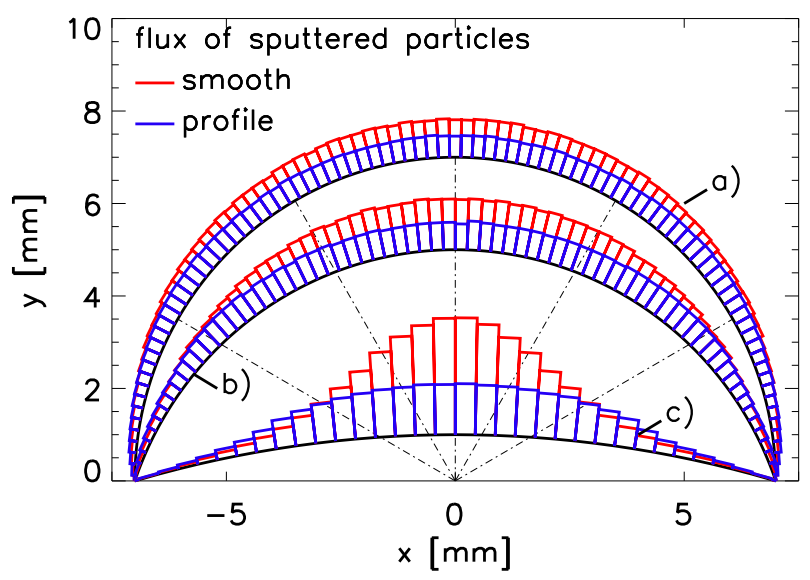

(b)

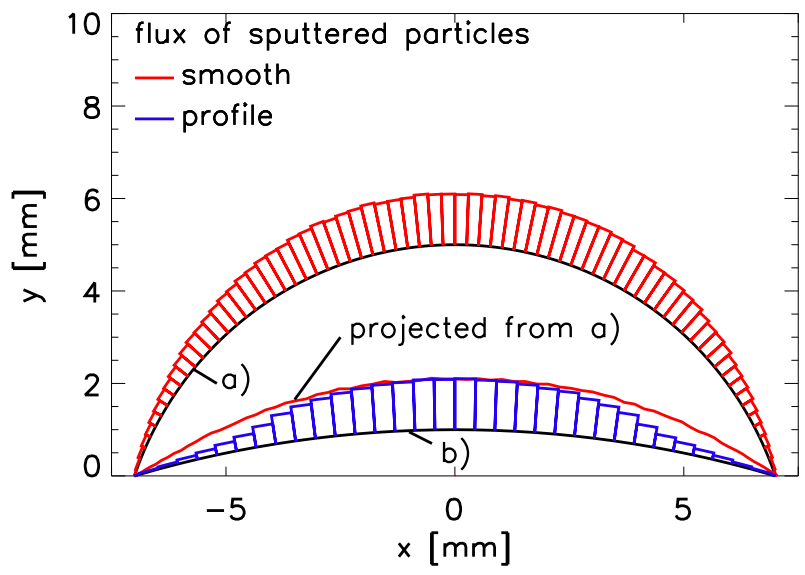

Fig. 12. (a) Spatial profile of the sputtered flux at various arc shaped diagnostics surfaces kept at $y=(7,5,1) \mathrm{mm}$. (b) projection of sputtering profiles from smooth (case b) onto an eroded surface (case c).

current.

Fig. 12a shows the spatial profile of the sputtered particles measured at the arc-shaped diagnostic surfaces kept at various heights above the $\mathrm{Cu}$ target for a smooth (flat) and a eroded well-shaped $\mathrm{Cu}$ target. This is done to study the effect of the relative downwards shift of the primary source of metal atoms in the cluster source. Arc-shaped diagnostic surfaces are chosen to include the particles which sputtered at larger angles and eventually lost to the side walls of the chamber. In Fig. 12a the labels $(a, b, c)$ correspond to the location of the diagnostic surface arc at $x=0$ and $y=(7,5,1) \mathrm{mm}$ respectively. The size of the bins just represents the resolution of the data-set for the plot. As one moves away from the initial target surface location the sputtered flux per unit area changes, however, the total sputtered flux remains the same. With increasing $r$, the variation in the sputtered flux profiles becomes smaller. In case (c) the sputtering profiles of the flat and eroded target are almost identical, even though the exact value for the eroded target is lower. This shows that the $1 / r^{2}$ dependence of the sputtered flux is quite prominent. Fig. 12b shows the sputtered flux profile from a (a) smooth target kept $\mathrm{y}=5 \mathrm{~mm}$ and diagnostic arc at $\mathrm{r}=0 \mathrm{~mm}$ and (b) eroded target with groove depth of $3.8 \mathrm{~mm}(\mathrm{y}=1.2$ $\mathrm{mm}$ ) diagnostic arc at $\mathrm{r}=5 \mathrm{~mm}$. In both these cases the 16 relative separation between the 
primary source of sputtered particles and the diagnostic surface is $4 \mathrm{~mm}$. If the profile of case (a) is projected onto case (b), it is clearly seen that the major sputtering contribution which comes from the center of the well in case of eroded profile, is identical to the once for a flat surface. Therefore, the significant parameter is the groove depth and it defines the location of the primary source. A correct estimate of the number density of the free atoms in the aggregation chamber can only be done by taking into account the downwards shift of the primary source locations and the positioning of the aggregation chamber relative to it. With the increasing depth of the primary source, the effective length of the aggregation region also increases, which in turn influences the cluster formation dynamics, cluster size and cluster intensity. The difference in the sputtering profiles in the tails of the distribution reflects the decrease in the sputtering due to the redeposition.

\section{Conclusions}

The simulation was able to reproduce the sputtering of a flat $\mathrm{Cu}$ target in a $\mathrm{DC}$ magnetron resulting in the formation of a race-track or an eroded well shaped surface. The geometrical constraints at the well shaped eroded surface leads to the reduction in the sputtering yield. This is due to the redeposition of the low-energy sputtered $\mathrm{Cu}$ atoms which do not have enough energy to create further collision cascade and simply get stuck on the well surfaces. During the erosion process the well becomes deeper and the major contribution of the sputtered flux comes from the center of the well which acts as a primary source of the metal atoms in the cluster source. Due to the combined effect of relative downwards shifts of the primary source during the erosion and the $1 / r^{2}$ dependence of the sputtered flux, the density of the $\mathrm{Cu}$ atoms above the target decreases. This leads to the decrease in the number of $\mathrm{Cu}$ atoms needed for cluster formation in the aggregation region.

\section{References}

[1] I. Kolev and A. Bogaerts. J. Vac. Sci. Technol. A, 27(1):20, (2009). 
[2] M. Ganeva, A. V. Pipa, and R. Hippler. Surface ES Coating Technology, 213:41, (2012).

[3] V. N. Popok, I. Barke, E. E. Campbell, and K-H. M.-Broer. Surf. Sci. Rep., 66:347, (2011).

[4] Klaus Ellmer. Magnetron discharges for thin film deposition. In Rainer Hippler, Holger Kersten, Martin Schmidt, and Karl H. Schoenbach, editors, Low Temperature Plasmas, pages 675-714. Wiley-VCH, Berlin, 2008.

[5] M. Ganeva, T. Peter, S. Bornholdt, H. Kersten, T. Strunskus, V. Zaporojtchenko, F. Faupel, and R. Hippler. Mass spectrometric investigations of nano-size cluster ions produced by high pressure magnetron sputtering. Contributions to Plasma Physics, 52(10):881-889, 2012.

[6] W. Eckstein A. Mutzke, R. Schneider and R. Dohmen. SDTrimSP: Version 5.00. IPP, Report, (12/8), 2011.

[7] A. Mutzke and W. Eckstein. Nucl. Instr. and Meth. B, 266:872, (2008).

[8] A. Mutzke and R. Schneider. IPP, Report, (12/4), 2009.

[9] W. Eckstein. Computer Simulation of Ion-Solid Interaction. Springer Series in Material Science, 10, 1991.

[10] Ivo Knittel, Marc Gothe, and Uwe Hartmann. Quantitative analysis of sputter processes in a small magnetron system. Journal of Vacuum Science 85 Technology A: Vacuum, Surfaces, and Films, 23(6):1714-1720, 2005.

[11] V.I. Kalikmanov. Classical nucleation theory. In Nucleation Theory, volume 860 of Lecture Notes in Physics, pages 17-41. Springer Netherlands, 2013.

[12] P. V. Kashtanov, B. M. Smirnov, and R. Hippler. Physics Uspekhi, 50(5):455, (2007).

[13] X.-Y.Liu, M.S. Daw, J.D. Kress, D.E. Hanson, V. Arunachalam, D.G. Coronell, C.-L. Liu, and A. F. Voter. Thin Solid Films, 422:141, (2002).

[14] J. P. Biersack and W. Eckstein. Appl. Phys. A, 34:73, (1984).

[15] M. Bloomfield and T. Cale. Mat. Res. Soc. Symp. Proc., 616:147, (2000).

[16] I. Kolev and A. Bogaerts. J. Appl. Phys., 104(1):093301, (2008). 


\section{List of Figures}

1 Schematics of the magnetron discharge field configuration.

2 General schematic of the magnetron cluster source. A circular $\mathrm{Cu}$ target of 2 " in diameter is used as cathode which is biased by a negative DC voltage and the anode is grounded. The anode-cathode distance is $0.3 \mathrm{~mm}$. The photo shows a magnified cross-section of the race-track profile.

3 Experimentally measured total cluster current as a function of target lifetime.

4 Schematics of the 1D and 2D target geometry in SDTrimSP.

5 Evolution of the surface. The experimental (red region) and simulated (yellow curve) target surface morphology as a function of fluence is shown.(a)-(e) the fluence is $(0,20,40,60,80) \times 10^{24} \mathrm{Ar}^{+}$ions $/ \mathrm{m}^{2}$.

6 Target groove depth and the total sputtering yield as a function of lifetime.

$7 \quad$ Profile and direction of the incident $A r^{+}$ions (black curves and arrows respectively), corresponding sputtered flux envelop and the profile of the sputtered flux (red curve) for an ideal flat (a) and an eroded (b) target.

8 Angular distribution of the sputtered $\mathrm{Cu}$ atoms for different angle of incidence.

$9 \quad$ Energy distribution of the sputtered particles measured at the diagnostics surface held at $0 \mathrm{~mm}$ above the initial geometrical surface in y-direction for (a) flat surface and (b) for a surface with race-track profile.

10 Contribution of the four type of processes to the sputtering vs angle of incidence $\alpha$. 
11 Sputtered flux per unit length measured at various locations above the target.

12 (a) Spatial profile of the sputtered flux at various arc shaped diagnostics surfaces kept at $y=(7,5,1) \mathrm{mm}$. (b) projection of sputtering profiles from smooth (case b) onto an eroded surface (case c). 